\title{
Estudio Comparativo de Perfiles Metabólicos Minerales en Lecherías de dos Regiones de Nariño
}

\section{A comparative study of metabolic mineral profiles on dairy farms from two regions in Nariño department}

\author{
Darío A. Cedeño-Quevedo ${ }^{1 *}$, Alejandro Ceballos-Marquez ${ }^{2}$ \\ Catalina Garzón ${ }^{3 *}$, Carmen A. Daza-Bolaños ${ }^{4 *}$ \\ ${ }^{1} \mathrm{MSc}$, Facultad de Ciencias Pecuarias, Universidad de Nariño \\ ${ }^{2} \mathrm{MVZ}, \mathrm{PhD}$, Universidad de Caldas, Departamento de Producción Animal. \\ Manizales, Caldas, Colombia \\ ${ }^{3,4} \mathrm{MV}$, Facultad de Ciencias Pecuarias, Universidad de Nariño, \\ "Grupo de investigación Buiatria, Nariño, Colombia \\ *Email:dcedeno@udenar.edu.co
}

Recibido: Diciembre 12 de 2010. Aceptado: Abril 21 de 2011

\section{RESUMEN}

El presente trabajo tiene como objetivo evaluar la concentración de calcio $(\mathrm{Ca})$, fosforo $(\mathrm{P})$ y magnesia $(\mathrm{Mg})$ en sangre de vacas lecheras en el periodo seco, posparto y pico de la producción en dos regiones de altura en Nariño, Colombia. Se evaluaron 351 vacas Holstein de ganaderías ubicadas entre 2600 y $3200 \mathrm{msnm}$ de Pasto y Guachucal - Tuquerres. Los pastos que se encontraron en las praderas fueron: Kikuyo (Pennisetum clandestinum), Azul orchoro (Dactylis glomerata), Rye grass (Loliumsp), Falsa Poa - Saboya (Holcus lanatus), trébol rojo (Trifolium pratense) y trébol blanco (Trifolium repens). Los animales fueron divididos en tres grupos de acuerdo a su estado productivo (periodo seco, inicio y pico de lactancia). Se determinó la concentración de $\mathrm{Ca}, \mathrm{P}$ y Mg por colorimetría. Los datos fueron sometidos a estadística descriptiva. En Pasto los niveles de $\mathrm{Ca}$ $(1,94 \mathrm{mmol} / \mathrm{L})$ en los tres estados productivos tuvieron promedios inferiores al valor considerado como promedio referencial. En Guachucal-Tuquerres la concentración de Ca fue mayor (2,3 mmol/L). A diferencia de los resultados observados para $\mathrm{Ca}$, no se encontraron diferencias para la concentración de $\mathrm{P}(1,81 \mathrm{mmol} / \mathrm{L})$ y $\mathrm{Mg}$ $(1,03 \mathrm{mmol} / \mathrm{L})$ según la región, estado productivo o número de partos de la vaca $(P>0,05)$. Los resultados de este estudio permiten concluir que las concentraciones de fósforo y magnesio se mantuvieron dentro del rango referencial, lo que indica que en el periodo de transición en vacas clínicamente sanas, tanto el aporte como el mecanismo homeostático que regula su concentración sanguínea, son adecuados y permiten concentraciones séricas compatibles con un balance metabólico nutricional óptimo en la vaca. Sin embargo, los valores para calcio difieren entre la región de Pasto y Guachucal-Tuquerres, lo que refleja cambios en el manejo nutricional de las vacas. 
Palabras claves: Calcio, fosforo, magnesio, Holstein.

\section{ABSTRACT}

The present study was aimed at assessing the calcium $(\mathrm{Ca})$, phosphorus $(\mathrm{P})$ and magnesium $(\mathrm{Mg})$ concentration in the blood of dairy cows from two areas of the Nariño department, Colombia, during dry, postpartum and peak production periods; 351 Holstein cows were evaluated from herds grazing at 2,600 to 3,200 metres above sea level in Pasto and Tuquerres Guachucal. The types of pasture found on the farms were kikuyu (Pennisetum clandestinum), orchardgrass (Dactylis glomerata), rye grass (Lolium sp), Yorkshire fog (Holcus lanatus), red clover (Trifolium pratense) and white clover (Trifolium repens). The cows were divided into three groups according to their production state (dry period, beginning and peak lactation). Colorimetry was used for determining $\mathrm{Ca}, \mathrm{P}$ and $\mathrm{Mg}$ concentration in blood and descriptive statistics were used for presenting the results. Average $\mathrm{Ca}$ levels $(1.94 \mathrm{mmol} / \mathrm{L})$ in cows from Pasto regarding the three production states were considered to be below the average reference value; average Ca concentration $(2.3 \mathrm{mmol} / \mathrm{L})$ was higher in Guachucal-Tuquerres. Unlike the results observed for $\mathrm{Ca}$, no differences were found for $\mathrm{P}(1.81 \mathrm{mmol} / \mathrm{L})$ or $\mathrm{Mg}$ concentration $(1.03 \mathrm{mmol} / \mathrm{L})$ by region, production status or number of calvings $(p>0.05)$. This study's results thus led to concluding that phosphorus and magnesium concentrations came within the reference range, indicating that clinically-healthy cows' transition period (regarding both input and the homeostatic mechanism regulating their blood levels) were suitable and ensured serum concentrations compatible with optimal nutritional metabolic profiles and mineral balance in the cows in question. However, there were differences in calcium values regarding the Pasto and Guachucal-Tuquerres areas, reflecting changes in these cows' nutritional management.

Key words: Calcium, phosphorus, magnesium, Holstein.

\section{INTRODUCCIÓN}

El análisis de los perfiles metabólicos (PM) es un instrumento paraclinico que permite estudiar los trastornos metabólicos y obtener un acercamiento a la evaluación del balance nutricional del organismo (Macrae et al., 2006). Payne et al. en 1970 establecieron la metodología del uso de los perfiles metabólicos, habiéndose descrito también su uso en Colombia (Ceballos et al., 2002).

La baja producción láctea y los problemas de infertilidad, son las causas principales de los altos costos de producción en el ganado bovino lechero. Los desordenes nutricionales posparto se relacionan con el manejo nutricional durante el periodo seco, así los desordenes metabólicos puede ser minimizados con una apropiada dieta y correcto manejo nutricional durante esta etapa.

La transición entre el final de la gestación y el inicio de la lactancia en la vaca, ha sido definida como un período critico para el metabolismo y bienestar de la vaca, particularmente entre las tres últimas semanas de gestación y las tres primeras de lactancia (Guo et al., 2007) Así, un objetivo clave del manejo nutricional de la vaca en transición es la detección temprana de aquellas vacas que manifiestan un mayor grado de desequilibrios metabólicos con respecto a otras vacas en las mismas condiciones fisiológicas y de manejo. Estas vacas estarán más propensas a la presentación de enfermedades metabólicas, por lo que el uso de los PM para su detección puede ayudar a tomar las medidas necesarias para revertir el problema (Wathes et al. 2007).

Los minerales aunque se encuentran en los tejidos, deben ser suministrados en la ración dado que no pueden ser sintetizados por el organismo. Debe considerarse que un buen manejo de la nutrición mineral consiste en aportar la cantidad necesaria según los requerimientos de la vaca, lo que puede ser factible de evaluar mediante su determinación en diferentes tejidos en el animal y saber así, si el aporte es suficiente o no (Mc Donald et al. 1997). Entre las enfermedades metabólicas asociadas con desequilibrios minerales cabe destacar la 
hipocalcemia, trastorno que suele presentarse después del parto y que se caracteriza por parálisis muscular, y en casos graves, coma y muerte del animal (Lincoln y Lane 1997). La hipocalcemia es un factor predisponerte para la distocia, prolapso uterino, retención de placenta, desplazamiento del abomaso y cetosis (Shaver 1997). Es importante destacar que la hipocalcemia es causada por un desequilibrio entre el egreso de calcio en el calostro en relación con las reservas extracelulares, en conjunto con una inadecuada respuesta por parte de los tejidos a la acción de las hormonas reguladoras del metabolismo del calcio (Chagas et al. 2011).

De otra parte, la concentración foliar de magnesio no es suficiente para satisfacer el requerimiento de bovinos en pastoreo. Sandoval et al en 1998 y Martens et al en el 2000 argumentaron que un aporte insuficiente unido a los egresos por producción de leche, la interacción antagónica con

\section{MATERIALES Y METODOS}

La investigación fue realizada en ganaderías ubicadas en zonas de altura comprendidas entre 2600 y 3200 msnm en las regiones de Pasto Guachucal - Tuquerres. El sistema productivo de los hatos es clasificado como lechería especializada en pastoreo intensivo más suplementación. La raza predominante es la Holstein con una producción promedio de 26 litros/ vaca/día, El número de ordeños por día es de dos en todos los predios.

Los pastos que se encontraron en las praderas fueron: Kikuyo (Pennisetum clandestinum), Azul orchoro (Dactylis glomerata), Rye grass (Lolium $\mathrm{sp}$ ), Falsa Poa - Saboya (Holcus lanatus), trébol rojo (Trifolium pratense) y trébol blanco (Trifolium repens). Los pastos se utilizan bajo el sistema de pastoreo directo rotacional. Se tomaron muestras de forraje donde las vacas iban a pastorear, se embalaron en bolsas, se rotularon y se enviaron al laboratorio especializado de la Universidad de Nariño para el estudio bromatológico. otros minerales y por la presencia de factores que interfieren su absorción, puede causar deficiencias de magnesio especialmente en el inicio de la lactancia. Por otro lado la los niveles altos de potasio puede llevar ovulaciones retardadas, un desarrollo menor del cuerpo luteo y aumentar la incidencia de anestro (Weiss 2004). Por lo anterior, el final del período seco así como el inicio de la lactancia en la vaca, se caracterizan por una serie de alteraciones en el metabolismo mineral donde la hembra está obligada a adaptar su metabolismo para mantenerse en equilibrio y disminuir los factores asociados con la presentación de enfermedades metabólicas (Overton y Waldron 2004).

En consideración a lo anterior, el objetivo de este trabajo es presentar algunos resultados preliminares de la evaluación metabólica mineral en vacas en fase de transición en dos regiones geográficas del departamento de Nariño.

Los animales son suplementados con alimentos balanceados según su producción. En todas las fincas se suministra sal mineralizada a voluntad, con un contenido promedio de calcio, fósforo y magnesio era de $14.0 \%, 8.0 \%$ y $0.3 \%$, respectivamente. El suministro de agua es permanente y a voluntad.

En total se evaluaron 148 vacas en 12 fincas de la región de Pasto y 203 vacas en 13 explotaciones de Guachucal-Túquerres. De acuerdo a las etapas de mayor demanda metabólica en la fase de producción, los animales de cada finca fueron divididos en tres grupos. El primer grupo comprendió vacas en periodo seco; 20 días preparto, el segundo grupo animales en inicio de lactancia ( 0 a 20 días en leche) y el tercer grupo vacas en la etapa de pico de producción (90 a 150 días gen leche). El rango de partos de las vacas fluctuó entre 1 y 14. Los animales que se escogieron para el estudio estaban aparentemente sanos y sin presencia de signos de alteraciones sanitarias o nutricionales. 
Las muestras de sangre se tomaron en la tarde, después del ordeño con el fin de evitar variaciones en las actividades diarias y estrés innecesario en los animales. Las muestras fueron tomadas por venopunción coccígea sin anticoagulante. El suero fue obtenido por centrifugación a $1500 \mathrm{rpm}$ por 5 minutos. A partir del suero, por medio de espectrofotometría luz visible o UV se determinaron los niveles séricos de los perfiles metabólicos. Los resultados se expresaron en unidades estándar internacionales (SIU).

Los parámetros estadísticos comprendieron pruebas de normalidad distributiva, valores promedio $(\mathrm{X})$, desviación estándar (DE), rango e intervalo de confianza (IC) al $95 \%$, además el coeficiente de variación (CV) para cada variable. Se fijó un nivel de significancia $p<0.05$. Las

\section{RESULTADOS}

Para las vacas del estudio de las dos regiones de Nariño el rango de partos de fluctuó entre 1 y 14 , con un promedio de $3,0 \pm 1,8$. La producción de leche comparaciones entre grupos se realizaron mediante análisis de varianza, determinando como fuentes de variación, la etapa productiva en la cual se encontrara la vaca. Se fijó un nivel de significancia $\mathrm{p}<0.05$ (Zar 1996). Se obtuvo la frecuencia de presentación de predios con grupos de vacas que presentaron valores promedio para calcio, fósforo y magnesio inferiores o superiores al valor promedio referencial citado por Kaneko et al (1997) y Wittwer et al (1993), quienes señalan los siguientes rangos como referenciales para cada metabolito analizado, calcio: $2.0-2.6 \mathrm{mmol} / \mathrm{L}$, fósforo: $1.1-2.3 \mathrm{mmol} /$ $\mathrm{L}$, y magnesio: $0.7-1.1 \mathrm{mmol} / \mathrm{L}$. En los casos en que el ANOVA resultó significativo $(p<0,05)$, se aplicó la prueba de comparación de medias (Tukey). Los análisis estadísticos se efectuaron con la ayuda de un programa informático en Stata v. 10.1 (Stata Corp. Collage Satation, Tx, USA).

fue en promedio $22,0 \pm 7,2 \mathrm{~kg} / \mathrm{vaca} / \mathrm{día}$. La condición corporal de las vacas fue en promedio $3,3 \pm 0,4$. El promedio y desviación estándar para cada región esta descrito en la tabla 1.

Tabla 1. Promedio y desviación estándar de partos, producción de leche y condición corporal en vacas de lecherías de Pasto y Guachucal-Túquerres, Nariño

\begin{tabular}{lccccc}
\cline { 3 - 5 } \multicolumn{1}{c}{ Materia Prima } & MS (\%) & PB & EE & CZ & EB \\
(Kcal/Kg)
\end{tabular}

El análisis bromatológico para los pastos está descrito en la tabla 1. Los resultados son validos para la época (seca) en que se tomaron y el porcentaje corresponde al porcentaje de materia seca.

El rango, el promedio y la desviación estándar para la concentración de calcio, fósforo y magnesio en vacas lecheras de hatos de la cuenca lechera de Nariño se presentan en la tabla 2. La concentración de calcio presentó variaciones según el estado productivo y con relación al momento del parto; así, en las vacas del municipio de Pasto se presentaron variaciones entre el periodo preparto $y$ etapa posparto, $(p<0.05)$, mientras que en las vacas de la región de Guachucal-Tuquerres no se encontraron variaciones para el mismo mineral.

No se presentaron grupos de vacas con valores de fósforo inferiores a $1.1 \mathrm{mmol} / \mathrm{L}$. No obstante, fueron encontrados predios localizados en la región de Guacucal - Tuquerres con grupos de vacas con una fosfatemia promedio superior a $2.6 \mathrm{mmol} / \mathrm{L}$,

La concentración de magnesio no presentó diferencias significativas entre los grupos estudiados, según la etapa productiva $(p>0.05)$. 
Igualmente, no se observaron en los grupos de vacas, tanto en preparto como en postparto, con valores promedio por fuera del rango señalado como referencial para la concentración de magnesio en bovinos.

Tabla 2. Concentración (\% en base seca) de proteína cruda, calcio, fósforo y magnesio observada en especies forrajeras de fincas lecheras del trópico alto del departamento de Nariño

\begin{tabular}{lllll}
\hline \multicolumn{1}{c}{ Especie forrajera } & $\begin{array}{c}\text { Proteína } \\
\text { cruda }\end{array}$ & $\mathbf{C a} \%$ & $\mathbf{P} \%$ & $\mathbf{M g \%}$ \\
& 14.63 & 0.42 & 0.28 & 0.28 \\
\hline Kikuyo (Pennisetum clandestinum) & 23,19 & 1,13 & 0,37 & 0.31 \\
Trébol blanco (Trifolium repems) & 25,30 & 1.01 & 0.29 & 0.45 \\
trébol rojo (Trifolium pratense) & 11.95 & 0.30 & 0.25 & 0.07 \\
Falsa Poa-Saboya(Holcus lanatus) & 21.6 & 0.28 & 0.26 & 0.24 \\
Rye grass (Lolium sp) & 11,6 & 0.45 & 0.29 & 0.16 \\
Azul orchoro (Dactylis glomeratd) & & & & \\
\hline
\end{tabular}

Laboratorio de bromatología de la Universidad de Nariño

El rango, el promedio y la desviación estándar para la concentración de calcio, fósforo y magnesio en vacas lecheras de hatos de la cuenca lechera de Nariño se presentan en la tabla 2. La concentración de calcio presentó variaciones según el estado productivo y con relación al momento del parto; así, en las vacas del municipio de Pasto se presentaron variaciones entre el periodo preparto $y$ etapa posparto, $(p<0.05)$, mientras que en las vacas de la región de Guachucal-Tuquerres no se encontraron variaciones para el mismo mineral.

No se presentaron grupos de vacas con valores de fósforo inferiores a $1.1 \mathrm{mmol} / \mathrm{L}$. No obstante, fueron encontrados predios localizados en la región de Guacucal - Tuquerres con grupos de vacas con una fosfatemia promedio superior a $2.6 \mathrm{mmol} / \mathrm{L}$,

La concentración de magnesio no presentó diferencias significativas entre los grupos estudiados, según la etapa productiva $(p>0.05)$. Igualmente, no se observaron en los grupos de vacas, tanto en preparto como en postparto, con valores promedio por fuera del rango señalado como referencial para la concentración de magnesio en bovinos.

En general los valores promedio de Ca en las vacas de la región de Pasto fueron inferiores en los tres estados productivos al valor considerado como promedio referencial, 2,3 $\mathrm{mmol} / \mathrm{L}$ (Kaneko et al.1997). Es clara una mayor concentración de Ca en las vacas de la zona de Guachucal-Túquerres $(P<0,05)$, no hubo diferencias $(P>0,05)$ según el estado productivo y el número de partos.

A diferencia de los resultados observados para $\mathrm{Ca}$, no se encontraron diferencias para la concentración de $\mathrm{P}$ y Mg según la región, estado productivo o número de partos de la vaca $(P>0,05)$. No obstante, sí se observaron algunos valores para $P$ superiores al límite superior de referencia $(2,3 \mathrm{mmol} / \mathrm{L})$, donde posiblemente el consumo de suplementos minerales altos en $\mathrm{P}$ unido a un contenido de $\mathrm{P}$ alto en el pasto, sea la causa de la elevación de la concentración sérica de fósforo (Brokman 2008). 
Tabla 3. Concentración de $\mathrm{Ca}, \mathrm{P}$ y Mg sérico según el estado productivo en vacas de lecherías de Pasto y Guachucal-Túquerres, Nariño (promedio y desviación estándar)

\begin{tabular}{lccc}
\hline & \multicolumn{3}{c}{ Estado productivo } \\
\hline \multicolumn{1}{c}{ Pasto } & Preparto & Inicio de lactancia & Lactancia \\
$\mathrm{Ca}^{*}$ & $1,94 \pm 0,4^{\mathrm{a}}$ & $1,90 \pm 0,3^{\mathrm{a}}$ & $2,00 \pm 0,3^{\mathrm{b}}$ \\
$\mathrm{P}$ & $1,84 \pm 0,6$ & $1,80 \pm 0,6$ & $1,70 \pm 0,5$ \\
$\mathrm{Mg}$ & $1,10 \pm 0,1$ & $1,00 \pm 0,1$ & $1,00 \pm 0,1$ \\
GuachucalTúquerres & & & \\
$\mathrm{Ca}$ & $2,43 \pm 0,5^{\mathrm{b}}$ & $2,43 \pm 0,5^{\mathrm{b}}$ & $2,44 \pm 0,5$ \\
$\mathrm{P}$ & $1,83 \pm 0,5$ & $1,85 \pm 0,5$ & $1,86 \pm 0,5$ \\
$\mathrm{Mg}$ & $1,00 \pm 0,1$ & $1,03 \pm 0,1$ & $1,02 \pm 0,1$ \\
\hline
\end{tabular}

${ }^{\star} \mathrm{mmol} / \mathrm{L}$. Cifras con letras distintas entre filas o columnas significan diferencia significativa $(P<0.05)$

\section{DISCUSIÓN}

Los valores observados para los metabolitos descritos en las zonas de Pasto y GuachucalTúquerres, Nariño, son valores similares a otros constatados en lecherías de la zona del Viejo Caldas por Ceballos et al en el 2004. Los que también se han caracterizado por presentar una alta variación en la población y reflejando diferencias en el manejo nutricional de los animales según las explotaciones.

Particularmente el $\mathrm{Ca}$, está regulado bajo un estricto control homeostático que trata de mantener sus concentraciones dentro de valores considerados; por lo tanto, su inclusión en el PM sólo tendría utilidad para conocer si la capacidad homeostática está funcionando adecuadamente y no como un reflejo del aporte en la ración. EI P, K, Mg y S son algunos minerales cuya concentración plasmática es más sensible a los cambios en su contenido en la ración como lo determinó Van Saun (1997).

La concentración sérica promedio de calcio en vacas de la región de Guachucal - Tuquerres se encontró dentro del rango reportado por la literatura (Roussel et al. 1997) como referencial. El periparto de la vaca lechera, en especial el período que ha sido denominado de transición, se caracteriza por cambios endocrinos que buscan mantener los niveles séricos de calcio dentro de un estrecho margen (Guo et al. 2007), lo que está reflejado en una variación mínima en la concentración promedio del mineral en los grupos de vacas y en los períodos productivos estudiados.

Se presentan diferentes mecanismos homeostáticos que tratan de mantener la calcemia dentro de un margen estrecho, con el objeto de evitar la hipocalcemia repentina que caracteriza el inicio de la lactancia, dentro de estos mecanismos se tiene la adaptación de la absorción intestinal regulada en parte por el flujo de alimentos al intestino (Lincoln y Lane 1990); el aumento de la resorción ósea, mediado por la hormona paratiroidea (PTH), y el aumento de la 1,25 dihidroxicolecalciferol (vitamina D3) con el consecuente mejoramiento de la absorción intestinal ((Kronqvist et al 2011). 
No obstante, aunque el calcio cuenta con estos mecanismos de regulación homeostática, sus niveles séricos también pueden verse afectados por la concentración del mineral en la dieta (Szcensi et al. 1994 y Van Saun et al. 1997). Pese a lo anterior, la concentración sérica de calcio presentó diferencias según el estado productivo en las vacas de la región de Pasto. Lo anterior está relacionado con la inestabilidad de los mecanismos encargados de la regulación metabólica de la calcemia en los primeros días después del parto, caracterizados por una hipocalcemia repentina que alcanza su punto crítico en el día 10 después del parto, pese a que la vaca puede presentar valores séricos de PTH y vitamina D3 adecuados. Se ha señalado que una posible causa para este fenómeno es la incapacidad de la vaca para regular la calcemia por la presencia de factores como un bajo consumo de magnesio y la alta concentración de potasio en los forrajes disponibles para las vacas en pastoreo (Overton et al. 2004), como es el caso en las vacas de Pasto, donde las praderas son muy abonadas. La interacción antagónica con otros minerales y por la presencia de factores que interfieren su absorción, puede causar deficiencias de magnesio especialmente en el inicio de la lactancia.

El promedio de la concentración de fósforo fue similar a los valores reportados en otros estudios realizados. Guarda relación con un mayor egreso del mineral a través de la leche y a un requerimiento mayor en las vacas con producciones elevadas comparado con vacas de producciones inferiores como lo reportan en las tablas del National Research

\section{CONCLUSIONES}

Se considera como grupo de riesgo las vacas en el período seco, lo que obliga a tomar medidas preventivas para que la vaca llegue en óptimas condiciones al parto. Al igual que las vacas que se encuentran en el inicio de la lactancia, donde es importante evaluar la capacidad de adaptación del animal a un nuevo estado productivo.

Las regiones de Pasto y Guachucal-Túquerres presentan, en promedio, valores para los diferentes
Council (2001). Una mayor variación en la concentración sérica de algunos indicadores bioquímicos nutricionales (macrominerales), es un reflejo de la acción de diversos factores que pueden inducir cambios en su concentración sérica, lo que significa que el indicador tiene entonces un menor grado de regulación homeostática y reflejaría, en mejor forma, el balance nutricional comparado con otros indicadores regulados homeostáticamente (calcio). Estos resultados son coincidentes con reportes de Goff (2003) donde se señala que la concentración sérica de fósforo puede variar por diversos factores; entre otros, según el estado productivo, la cantidad de leche producida y el contenido del mineral en los alimentos.

La concentración de magnesio no presentó diferencias en cuanto al estado productivo en las vacas. Los valores promedio fueron concordantes con los reportados en otros estudios (Kronqvist et al. 2011), además estaban dentro del rango referencial para este mineral (Wittwer 1993). Los promedios observados en los grupos eran compatibles con una concentración adecuada en la dieta; no obstante, las vacas de la mayoría de las explotaciones lecheras están expuestas a la presencia de diferentes factores que interfieren con la absorción del magnesio, coeficiente que de por sí es bajo en rumiantes adultos; entre otros factores, se tiene la deficiencia de carbohidratos fermentables, el exceso en la concentración de nitrógeno no proteico en los forrajes, y uno de los factores que más incide sobre el metabolismo del magnesio es el exceso de potasio y fósforo de la dieta (Sandoval et al. 1998).

metabolitos evaluados en un perfil metabólico en vacas productoras de leche similares a los observados en otras regiones del país. Sin embargo, la región juega un papel importante como una fuente de variación en los resultados, lo que refleja cambios en el manejo nutricional de las vacas. Como era de esperarse, para algunos metabolitos factores como el número de partos y el estado fisiológico de la vaca son fuentes de variación a considerar en la interpretación de perfiles metabólicos. 
La concentración mineral de los forrajes estaría determinando en parte su aporte en las vacas objeto del estudio, influyendo así en las concentraciones observadas en sangre ya que el forraje es la principal fuente nutricional de los bovinos en estos sistemas productivos basados en pastoreo.

Los valores observados, tanto elevados como disminuidos, constituye una indicación que hay presencia de alteraciones que son un riesgo para la

\section{RECOMENDACIONES}

Es necesario realizar en estudios posteriores una evaluación de otros indicadores del metabolismo del magnesio, ya que pese a observar valores dentro de la referencia, se sabe por observaciones no publicadas que en las lecherías de la región de la cuenca lechera de Nariño, hay factores que interfieren con su metabolismo, como son la deficiencia de energía, el exceso de nitrógeno no proteico y el contenido de fósforo y potasio de los forrajes.

\section{AGRADECIMIENTOS}

Los autores expresan su agradecimiento a los estudiantes que integran el grupo de investigación de BUIATRIA del programa de Medicina veterinaria de la universidad de Nariño por el apoyo en el

\section{REFERENCIAS}

Brokman AM, Lekmkuhler JW, Undersander DJ. Reducing phosphorus inputs for grazing Holstein steers. J Aim Sci. 2008; 86: 712-719.

Ceballos A, Villa NA, Betancourt TE, Roncancio DV. Determinación de la concentración de calcio, fósforo y magnesio en el periparto de vacas lecheras en Manizales, Colombia. Rev Col Cienc Pec. 2004; 17(2):125-134.

Ceballos A, Villa NA, Bohórquez A, Quiceno J, Jaramillo M, Giraldo G. Análisis de los resultados de perfiles metabólicos en lecherías del trópico alto del eje cafetero colombiano. Rev Col Cienc Pec. 2002; 15:26-35. presentación de afecciones de diversa índole, especialmente enfermedades de la producción.

Los resultados bajo las condiciones en que fue realizado, permiten concluir que las concentraciones de fósforo y magnesio se mantuvieron dentro del rango referencial, lo que indica que en el periparto en vacas clínicamente sanas, tanto el aporte como el mecanismo homeostático que regula su concentración sanguínea, son adecuados y permiten concentraciones séricas compatibles con un balance metabólico nutricional óptimo en la vaca.

La concentración de fósforo en algunos grupos presentó valores elevados que señalan un aporte superior al requerimiento según el estado productivo de la vaca, lo que indica la necesidad de realizar estudios posteriores que permitan determinar cuál es la magnitud del exceso en el consumo y su efecto sobre el metabolismo de otros minerales, sobre la producción y fertilidad de la vaca, y en el medio ambiente.

trabajo de campo. Igualmente a la Vicerrectoria de Investigaciones, postgrados y relaciones internacionales (VIPRI) de la Universidad de Nariño por el aporte económico.

Goff JP, Horst RL. Milk fever control in the United States. Acta Vet Scand Suppl. 2003; 97: 145-147.

Guo J, Peters RR, Kohn RA. Effect of a transition diet on production performance and metabolism in periparturient dairy cows. J Dairy Sci. 2007; 90:52475258.

Kaneko JJ, Harvey JW, Bruss ML. 1997. Clinical biochemistry of domestic animals, 5th ed, San Diego, Academic Press. 932p.

Kronqvist C, Emanuelson U, Sporndly R, Holtenius $\mathrm{K}$. Effects of prepartum dietary calcium level on 
calcium and magnesium metabolism in periparturient dairy cows. J Dairy Sci. 2011; 9: 1365-1373.

Lincoln SD, Lane VM. Serum lonized calcium concentration in clinically normal dairy cattle, and changes associated with calcium abnormalities. J Am Vet Med Ass. 1990; 97:1471-74.

Macrae AI, Whitaker DA, Burrough E, Dowell A, Kelly $\mathrm{JM}$. Use of metabolic profiles for the assessment of dietary adequacy in UK dairy herds. Vet Rec. 2006; 155: 655-661.

Martens $\mathrm{H}$, Schweigel M. Pathophysiology of grass tetany and other hypomagnesemias: implications for clinical management. Vet Clin North Am: Food Anim Pract. 2000; 16: 339-68.

Mc Donald P, Edwards RA, Greenhalgh JFD, Morgan CA. 1997. Nutrición Animal, 5ª ed, Zaragoza, España. Editorial Acribia. 395p.

National Research Council. 2001. Nutrient requirements of dairy cattle, 7 th ed, Washington DC, National Academy Press. 381p.

Overton TR, Waldron MR. Nutritional Management of Transition Dairy Cows: Strategies to Optimize Metabolic Health. J Dairy Sci. 2004; 87: E105-E119.

Payne JM. 1989. Metabolic and nutritional diseases of cattle, Oxford, UK, Blackwell Scientific Publications. 149p.
Roussel AJ, Whitney MS, Cole DJ. Interpreting a bovine serum chemistry profile: part 2. Vet Med. 1997; 92:559-566.

Sandoval GL, Dellamea S, Pochon DO, Campos MV. Calcio, fósforo, magnesio y fosfatasa alcalina en vacas lecheras de una región subtropical suplementadas con óxido de magnesio. Rev Vet Méx. 1998;29:131-36.

Shaver RD. Nutritional risk factors of left displaced abomasum in dairy cow: A review. J Dairy Sci. 1997; 80:2449-2453.

Szenci O, Chew BP, Brydl E. Total ionized calcium in parturient dairy cows and their calves. J Dairy Sci. 1994; 77:1100-05.

Van Saun R. Nutritional profiles: a new approach for dairy herds. Bov Practition. 1997; 31: 43-50.

Wathes DC, Bourne N, Cheng Z, Mann GE, Taylor VJ, Coffey MP. Multiple correlation analyses of metabolic and endocrine profiles with fertility in primiparous and multiparous cows. J Dairy Sci. 2007; 90:1310-1325.

Weiss WP. .Macromineral digestion by lactating Dairy cows; factors affecting digestibility of magnesium. J Dairy Sci. 2004; 87;2167-2171.

Wittwer F, Heuer G, Contreras PA, Böhmwald H. Valores bioquímicos clínicos sanguíneos de vacas cursando con decúbito en el sur de Chile. Arch Med Vet. 1993; 25: 83-88.

Zar JH.1996. Biostatistical analysis, 3d ed, Upper Saddle River, USA, Prentice Hall. 662p. 